Gynäkologe 2013 · 46:440-441

DOI 10.1007/s00129-012-3117-3

Online publiziert: 19. Juni 2013

(c) Springer-Verlag Berlin Heidelberg 2013

T. Dimpfl ${ }^{1}$ H. Kölbl ${ }^{2} \cdot$ R. Tunn ${ }^{3} \cdot$ N. Maass ${ }^{4}$

${ }^{1}$ Frauenklinik, Klinikum Kassel

2 Universitätsklinik für Frauenheilkunde, Medizinische Universität Wien

${ }^{3}$ Deutsches Beckenbodenzentrum, St.-Hedwig-Krankenhaus, Berlin

${ }^{4}$ Frauenklinik für Gynäkologie und Geburtsmedizin, Universitätsklinikum Aachen

\title{
Urogynäkologie im Wandel
}

\section{Vom Stiefkind zum Hauptdarsteller}

Die Prävalenz von Deszensus und Inkontinenz einer immer älter werdenden Gesellschaft ist gut belegt. Ab einem Alter von 65-70 Jahren leidet statistisch jede zweite Frau unter entsprechenden Symptomen. Die demographische Prognose geht davon aus, dass im Jahr 2050 20\% der Bevölkerung älter als 65 Jahre sind. Dadurch ergibt sich ein enormer Versorgungsauftrag.

\section{\) Im Bereich der Urogynäkologie wird der Versorgungsauftrag weiter anwachsen}

Der Untertitel „Vom Stiefkind zum Hauptdarsteller“ beschreibt die Entwicklung der Urogynäkologie in Deutschland in den letzten 20 Jahren. Katalysiert durch die Entwicklung und Erfindung des TVT („tension free vaginal tape“, spannungsfreies Vaginalband) durch Ulf Ulmsten zu Beginn der 1990er-Jahre entwickelte sich die Urogynäkologie rasant weiter. Das einfache und klare Gedankenmodell und die ebenso klar definierte Operationsmethode löst eine Vielzahl von mehr oder weniger erfolgreichen Operationsmethoden ab. Durch die intensive Beschäftigung mit dieser Thematik, teils auch getriggert durch das verstärkte Engagement und Interesse der Industrie, erleben die klassischen gy- näkologischen vaginalen Operationstechniken eine Renaissance. Dazu finden die abdominalen Deszensusoperationen, etwa die Sakrokolpopexie, weitere Verbreitung. Der Anfang dieses Jahrtausends ist geprägt durch den Einsatz von alloplastischem Material in Form von vorderen, hinteren oder kompletten vaginalen Netzen. Die Chancen und Risiken dieser neuen Technik werden in diesem Heft dargestellt.

$\mathrm{Zu}$ dieser Zeit formierte sich die wissenschaftliche Community in der AGUB (Arbeitsgemeinschaft für. Urogynäkologie und plastische Beckenbodenrekonstruktion) neu, und eine große Gruppe interessierter Wissenschaftler trieb die Erstellung und Aktualisierung der Leitlinien voran. Neben der notwendigen Interdisziplinarität wählten wir als erste Arbeitsgemeinschaft den Weg über die Grenzen von Deutschland hinaus und bezogen die Kollegen aus Österreich und der Schweiz ein. Das Resultat waren erstmals für den deutschsprachigen Raum Europas gültige und gemeinsam erstellte Leitlinien.

Auf dem Fundament der wissenschaftlichen Evidenz wurde als individuelles Qualifikationsmerkmal in enger Anlehnung an die DEGUM(Deutsche Gesellschaft für Ultraschall in der Medizin) -Qualifikation das AGUB-Zertifikat I-III ins Leben gerufen.

Durch die Vielzahl von Aktivitäten einer dynamischen Gruppe konnte die Arbeit der AGUB großes Interesse fin- den und wuchs und wuchs, von etwa 350 Mitgliedern in 2006 auf über 800 in 2013. Damit ist die AGUB neben der AGO (Arbeitsgemeinschaft Gynäkologische Onkologie) die größte Arbeitsgruppe in der DGGG (Deutsche Gesellschaft für Gynäkologie und Geburtshilfe) und konnte damit in der neuen Satzung auch einen festen Sitz im Vorstand behaupten.

Nach Schaffung der wissenschaftlichen Grundlage unseres Tuns, nach der Definition einer persönlichen Qualifikation sowie nach Einbringen der Aktivität in den Vorstand unserer Gesellschaft war der nächste Schritt die Entwicklung klarer Strukturvorgaben für interdisziplinäre Kontinenz- und Beckenbodenzentren. Dieser Prozess wurde nach 2-jähriger sehr konstruktiver Gemeinschaftsarbeit mit den Vertretern der wissenschaftlichen Fachgesellschaften der Deutschen Gesellschaft für Urologie, der Deutschen Gesellschaft für Allgemein- und Viszeralchirurgie sowie der Deutschen Gesellschaft für Koloproktologie mit der Zertifizierung von 10 Pilotzentren in 2012 abgeschlossen und steht derzeit interessierten Zentren offen.

In den folgenden Artikeln möchten wir Ihnen die verschiedenen Facetten der Urogynäkologie auf der wissenschaftlichen Grundlage der Leitlinien darstellen, erweitert und ergänzt durch wichtige persönliche Erfahrungen von Meinungsbildnern in unserem Fach. 


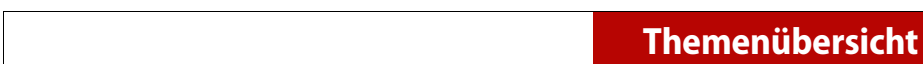

Wir wünschen Ihnen viele Anregungen und Diskussionen.

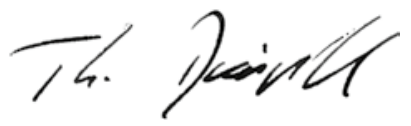

Prof. Dr. Thomas Dimpfl

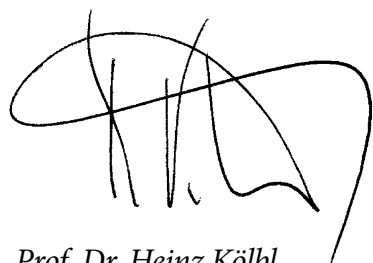

Prof. Dr. Heinz Kölbl

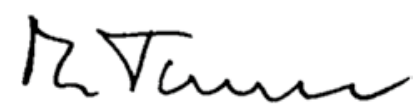

Prof. Dr. Ralf Tunn

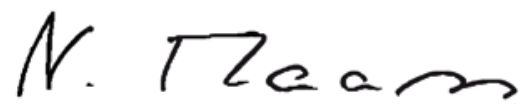

Prof. Dr. Nicolai Maass

\section{Korrespondenzadresse}

\section{Prof. Dr. T. Dimpfl}

Frauenklinik, Klinikum Kassel

Mönchebergstr. 41-43, 34125 Kassel

dimpfl@klinikum-kassel.de

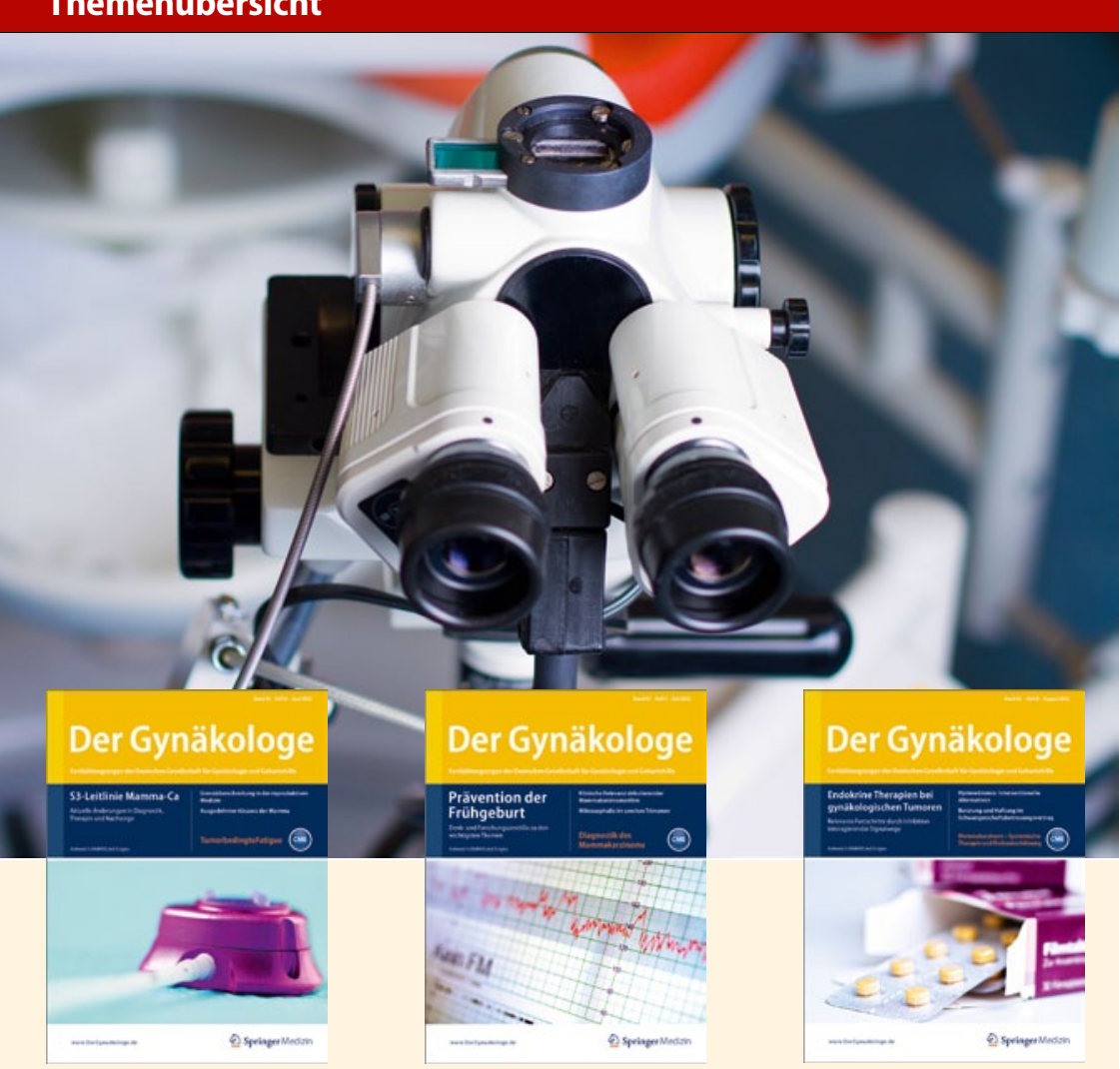

Der Gynäkologe bietet Ihnen jeden Monat umfassende und aktuelle Beiträge zu interessanten Themenschwerpunkten aus allen Bereichen der Gynäkologie und Geburtshilfe.

\section{2}

07/12 Prävention der Frühgeburt

08/12 Endokrine Therapien bei gynäkologischen Tumoren

09/12 Medizin für die Frau - Die Zukunft im Blick

10/12 Stellenwert der 3D-/4D-Sonographie

11/12 Organerhaltende Operationen

12/12 Onkologische Erkrankungen in der Schwangerschaft

\section{3}

01/13 Zukunft mit Kindern

02/13 Organisation der geburtshilflichen Versorgung

03/13 Hormontherapie - ein Update

04/13 Knochengesundheit

05/13 Schwangerschaft und Lifestyle

06/13 Biomarker

Änderungen vorbehalten
Möchten Sie ein bereits erschienenes Heft nachbestellen? Einzelne Ausgaben können Sie direkt bei unserem Kundenservice zum Preis von je EUR 36,- zzgl. Versandkosten beziehen:

So erreichen Sie unseren Kundenservice: Springer Customer Service Center GmbH Kundenservice Zeitschriften

Haberstr. 7,

69126 Heidelberg

Tel.: +496221 345-4303

Fax: +496221 345-4229

E-Mail: leserservice@springer.com 